\title{
Investigation of Starch Hydration by 2D Time Domain NMR
}

\author{
M. Witek ${ }^{a}$, H. Peemoeller ${ }^{b}$, J. Szymońska $^{c}$ \\ AND B. BlicharsKa ${ }^{a}$ \\ ${ }^{a}$ Institute of Physics, Jagiellonian University \\ Reymonta 4, 30-059 Kraków, Poland \\ ${ }^{b}$ Department of Physics, University of Waterloo \\ 200 University Avenue West, Waterloo, Ontario, Canada N2L 3G1 \\ ${ }^{c}$ Agriculture Academy, al. Mickiewicza 21, 31-120 Kraków, Poland \\ Proton exchange between spin groups of the solid matrix of hydrated \\ granular potato starch and water was studied using the $2 \mathrm{D}$ time domain \\ NMR. The proton spin-spin relaxation time $T_{2}$, and spin-lattice relaxation \\ time $T_{1}$ (selective and non-selective pulse sequences) were measured at room \\ temperature. The observed spin relaxation results were analysed for ex- \\ change assuming a two-site exchange model (between water and solid matrix \\ of starch). In this analysis we determined the intrinsic spin-lattice relaxation \\ time for water protons (49 ms) and solid starch matrix protons (172 ms), as \\ well as the water-starch magnetization exchange rate $\left(86 \mathrm{~s}^{-1}\right)$.
}

PACS numbers: 82.56.Na, 82.35.Pq

\section{Introduction}

The structure of native starch granules was studied using a variety of microscopic and scattering techniques, including optical, electron, and atomic force microscopy, light, X-ray, and neutron scattering [1-4]. The physical and structural properties of starch are strongly dependent on molecular interactions with water. NMR is an excellent technique for studying the state of water and its dynamic behaviour in polymeric materials $[5,6]$. In this paper, we report on a NMR investigation of the coupling between starch and water proton magnetizations in low hydrated starch sample.

\section{Materials and methods}

Granular potato starch, isolated in Nowamyl (Łobez, Poland) according to Polish Standard PN-A-74710, was dried in vacuum $\left(10^{-3}\right.$ Torr $)$, at $105^{\circ} \mathrm{C}$, for $24 \mathrm{~h}$ (dry sample). The potato starch hydrated sample was prepared by exposing the 
dry sample to $100 \%$ humidity in a glass desiccator for two days. The moisture content (MC) was $10.6 \%$, calculated as the weight of water relative to the dry wood weight, expressed in percent.

The measurements of $T_{1}$ and $T_{2}$ were performed using a pulsed WNS HB-65 NMR spectrometer working at $30 \mathrm{MHz} . T_{2}$ was obtained from the free-induction decay (FID), following a short $(1.7 \mu \mathrm{s}) 90^{\circ} \mathrm{RF}$ pulse. $T_{1}$ was measured using two different pulse sequences: non-selective standard inversion recovery sequence (hard-hard $T_{1}$ ), and the selective inversion recovery sequence (soft-hard $T_{1}$ ) with a soft $180^{\circ}$ pulse. The non-selective inversion recovery sequence $\left(180^{\circ}-\tau-90^{\circ}\right)$ uses short (time duration $=1.7 \mu \mathrm{s} \ll T_{2}$ ) pulses, or "hard" pulses, which invert all protons. In the selective sequence a low power $180^{\circ}$ pulse, applied for $60 \mu \mathrm{s}$ (a time greater than $T_{2}$ of the solid component magnetization), also called a "soft" pulse, is followed by a hard $90^{\circ}$ monitoring pulse. The soft $180^{\circ}$ pulse is only effective in inverting the water component magnetization. The second pulse rotates the total magnetization into the $x-y$ plane for detection. During the time $\tau$, Zeeman magnetization may exchange between spin groups. If the exchange rate is fast on the $T_{1}$ time scale a single $T_{1}$ is observed [7].

The 2D time domain NMR technique $([8,9]$ and references therein) was applied in the $T_{1}$ experiments. In this approach the data are acquired along the $t$ (FID time axis) and the $\tau$ axis, and stored in a 2D matrix with the indices representing the two time axes. Thus, for each value of $t$ a magnetization recovery curve is stored. The magnetization recovery curves at all values of $t$ were then simultaneously fit to a single exponential (for the hard-hard $T_{1}$ experiment) or a double exponential (for the soft-hard $T_{1}$ experiment) using the Marquardt non-linear least squares fitting algorithm. Such analysis of the $2 \mathrm{D}$ data set yields a $T_{1}$ for the single exponential case, and two $T_{1}$ values for the double exponential case. The $\tau=0$ intercepts, obtained from the fit for all $t$ values, are used to reconstruct a FID. By fitting the reconstructed FID to an appropriate FID function (Gaussian damped sinc for dry starch and a sum of Gaussian damped sinc and exponential for the hydrated starch) the apparent magnetization fractions (from the $t=0$ intercepts), and $T_{2}$ values of spin groups distinguished by values of $T_{1}$ are obtained.

\section{Results and discussion}

The FID for the hydrated sample of starch is shown in Fig. 1. In keeping with previous work [10] the presence of the small oscillation in the solid component of the FID prompts us to fit this component to a Gaussian damped sinc function. The liquid-like signal was modelled as an exponential so that the FID in the hydrated sample becomes,

$$
F_{s}(t)=f_{\mathrm{S}} \exp \left(-\left(\frac{t}{T_{2 \mathrm{G}}}\right)^{2}\right) \frac{\sin (a t)}{a t}+f_{L} \exp \left(-\frac{t}{T_{2 E}}\right),
$$

where $f_{\mathrm{S}}$ is the magnetization of the solid component, $T_{2 \mathrm{G}}$ is the spin-spin relaxation time associated with the Gaussian damping factor, $a$ is a constant, $f_{L}$ is the 


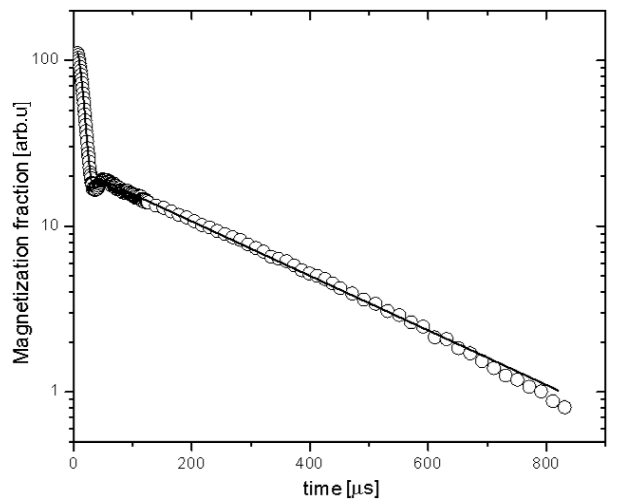

Fig. 1. Proton FID in the hydrated potato starch sample with $\mathrm{MC}=10.6 \%$. The solid line was calculated from Eq. (0) with fitted $T_{2}$ values and magnetization fractions given in Table.

TABLE

The observed relaxation parameters of potato starch sample hydrated to $10.6 \%$.

\begin{tabular}{c|c|c|c|c|c}
\hline \hline$T_{2}$ FID & Fraction [\%] & 82.2 & 17.8 & & \\
& $T_{2}[\mu \mathrm{s}]$ & $22.0 \pm 0.2$ & $264 \pm 2$ & & \\
\hline hard-hard $T_{1}$ & Fraction [\%] & 82.5 & 17.5 & & \\
& $T_{2}[\mu \mathrm{s}]$ & $22.4 \pm 0.3$ & $268 \pm 5$ & & \\
& $T_{1}[\mathrm{~ms}]$ & \multicolumn{2}{|c|}{$122 \pm 3$} & & \\
\hline soft-hard $T_{1}$ & Fraction [\%] & $(-) 16.2$ & 18.2 & 80.6 & 17.4 \\
& $T_{2}[\mu \mathrm{s}]$ & $21.7 \pm 1$ & $296 \pm 4$ & $22.9 \pm 0.4$ & $252 \pm 5$ \\
& $T_{1}[\mathrm{~ms}]$ & \multicolumn{2}{|c|}{$2.00 \pm 0.04$} & $120 \pm 3$
\end{tabular}

magnetization of the exponential component, and $T_{2 E}$ is its spin-spin relaxation time. The solid line (Fig. 1) was calculated from Eq. (0), with the $T_{2}$ values and normalized magnetization fractions, obtained from the fit, given in Table. It may be noted that the $T_{2}$ values obtained from the present fit of the Gaussian damped sinc function or sinc-Gaussian function $(\sim 20 \mu \mathrm{s})$ (Eq. (0)) are longer than those observed for the Gaussian fit for biological dry matrices $(\sim 14 \mu \mathrm{s})$ [8].

For the dry starch sample the main part of the FID (not shown) is well described by the sinc-Gaussian function with $T_{2 \mathrm{G}}=(21.1 \pm 0.1) \mu \mathrm{s}$. Therefore, in the FID of the hydrated sample the solid-like signal ( $82.2 \%$ of the signal), described by the sinc-Gaussian with $T_{2 \mathrm{G}}=(22.0 \pm 0.2) \mu \mathrm{s}$, was assigned to polymer protons. The remaining $17.8 \%$ of the signal with $T_{2 E}$ equal to $(264 \pm 2) \mu \mathrm{s}$, can be associated with protons of water.

In the analysis of the recovery curves from the hard-hard $T_{1}$ experiment a single $T_{1}$ was fitted. $2 \mathrm{D}$ time evolution analysis of these results yielded two 
reconstructed FIDs with the values of $T_{2}$ and fractions similar to those found in the FID experiments (Table). The fact that the magnetizations with liquid-like and solid-like $T_{2}$ 's exhibit the same value of $T_{1}$ shows that the exchange couples the spin-lattice relaxations of these two spin groups strongly enough to produce the single $T_{1}$.

More information about this system is obtained from the selective softhard $T_{1}$ experiment, in which we resolve two component magnetizations. The $T_{1}$ values and reconstructed FIDs obtained from the $2 \mathrm{D}$ analysis are shown in Fig. $2 \mathrm{a}$ and $\mathrm{b}$, respectively. The component magnetization $T_{1}$ values averaged over all time windows, the normalized magnetization fractions, and the reconstructed FID $T_{2}$ values are given in Table. The negative signal with short $T_{1}$ seen in the reconstructed FID (Fig. 2b) indicates the presence of magnetization exchange between solid-like and liquid-like spin groups.
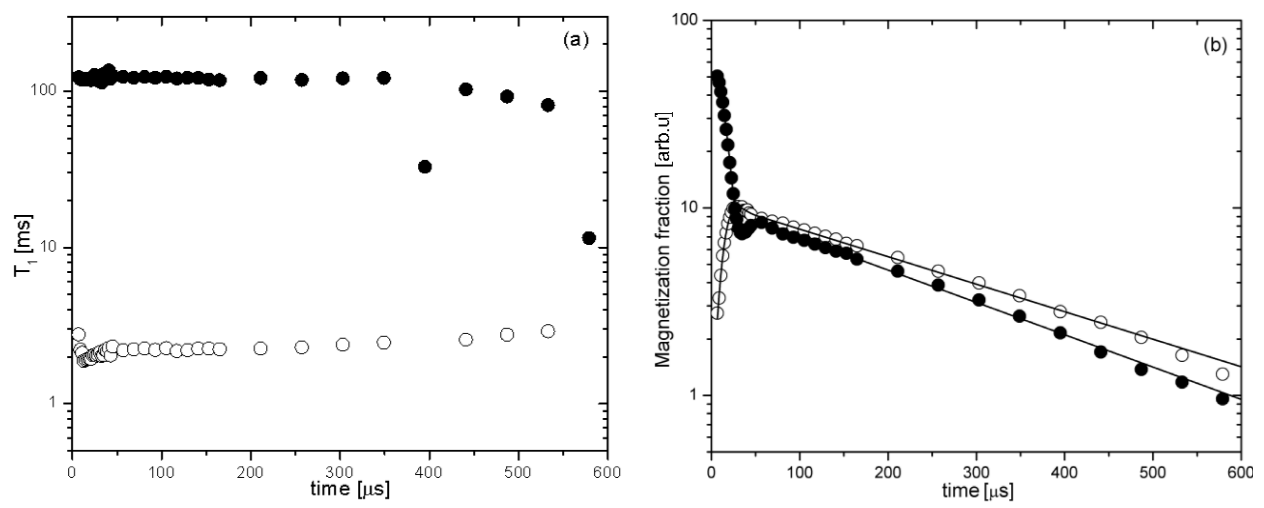

Fig. 2. Results of 2D time domain NMR experiment using the soft-hard $T_{1}$ sequence in hydrated potato starch sample at $30 \mathrm{MHz}$. (a) Variation of $T_{1}$ as a function of time window along FID: the magnetization recovery is characterized by two time constants ( $T_{1}$ 's) equal to $(2.00 \pm 0.04) \mathrm{ms}$ and $(120 \pm 3) \mathrm{ms}$ at all windows, (b) the reconstructed FIDs corresponding to the two component $T_{1}$ 's shown in (a).

The results in the hydrated potato starch sample have been interpreted in terms of a two-site exchange model, where the two sites correspond to the starch and water magnetization reservoirs. Letting the reduced magnetization of reservoir $i$ equal $m_{i}(\tau)=\left(M_{0 i}-M_{i}(\tau)\right) / 2 M_{0 i}$, its evolution in time within the two-site exchange model may be written,

$$
\frac{\mathrm{d} m_{i}(\tau)}{\mathrm{d} \tau}=-\left[\left(\frac{1}{T_{1, \text { int }}}\right)_{i}+k_{i j}\right] m_{i}(\tau)+k_{i j} m_{j}(\tau)
$$

where $(i, j)=($ starch, water $),\left(1 / T_{1, \text { int }}\right)_{i}$ is the intrinsic relaxation rate of the $i$ th reservoir magnetization, and $k_{i j}$ is the rate of magnetization transfer from the $i$ th to the $j$ th reservoir. The solution of Eq. (1) has the following form: 


$$
m_{i}(\tau)=C_{i}^{-} \exp \left(-\left(\frac{1}{T_{1, \text { app }}}\right)^{-} \tau\right)+C_{i}^{+} \exp \left(-\left(\frac{1}{T_{1, \mathrm{app}}}\right)^{+} \tau\right)
$$

where the $\left(1 / T_{1, \text { app }}\right)^{ \pm}$'s are the apparent relaxation rates, and the $C_{i}^{ \pm}$'s are apparent magnetization fractions, which are a function of the $\left(1 / T_{1, \text { int }}\right)_{i[j]}$ 's, $k_{i j}$ 's, intrinsic magnetization fractions, and effect of the preparation pulse in the inversion recovery sequence. The relations between apparent and intrinsic parameters are given by

$$
\begin{aligned}
& \left(\frac{1}{T_{1, \text { app }}}\right)^{ \pm}=\frac{1}{2}\left\{\left(\frac{1}{T_{1, \text { int }}}\right)_{i}+\left(\frac{1}{T_{1, \text { int }}}\right)_{j}+k_{i j}+k_{j i}\right. \\
& \pm \sqrt{\left.\left[\left(\frac{1}{T_{1, \text { int }}}\right)_{i}-\left(\frac{1}{T_{1, \text { int }}}\right)_{j}+k_{i j}-k_{j i}\right]^{2}+4 k_{i j} k_{j i}\right\}}, \\
& C_{i[j]}^{ \pm}= \pm \frac{m_{i[j]}(0)}{\left[\left(\frac{1}{T_{1, \text { app }}}\right)^{+}-\left(\frac{1}{T_{1, \text { app }}}\right)^{-}\right]} \\
& \times\left[\left(\frac{1}{T_{1, \text { int }}}\right)_{i[j]}-\left(\frac{1}{T_{1, \text { app }}}\right)^{\mp}+\left(1-\frac{m_{j[i]}(0)}{m_{i[j]}(0)}\right) k_{i j[j i]}\right],
\end{aligned}
$$

where $m_{i[j]}(0)$ are the reduced magnetizations of spins in the $i$ th or $j$ th site prior to period $\tau$.

In the experiment, the values of the magnetization fractions (values of $C_{i[j]}^{ \pm}$ in the model) are found from the reconstructed FIDs as shown in Fig. 2b (the values of parameters are given in Table). The experimental parameters, and model apparent parameters calculated from Eq. (3) for certain trial intrinsic values of these parameters are compared within an iterative minimization algorithm, giving the intrinsic relaxation parameters $\left(\left(1 / T_{1, \text { int }}\right)_{i[j]}\right.$ and $\left.k_{i j}\right)$, which represent the best match between these two sets of parameters [8]. We found the exchange rate $k_{\text {starch-water }}=86 \mathrm{~s}^{-1}$, and spin-lattice relaxation time $T_{1 \mathrm{starch}}=172 \mathrm{~ms}$ for the major $82.2 \%$ component, and $T_{1 \text { water }}=49 \mathrm{~ms}$ for the minor $17.8 \%$ component magnetization. It is seen that the obtained exchange rate is fast enough to satisfy the fast exchange condition, consistent with the observation of single-exponential $T_{1}$ in the hard-hard experiment.

The native potato starch granules are composed mainly of two glucose polymers: linear amylose and highly branched amylopectin. The molecular structure of native potato starch granules includes semi-crystalline layers (mainly built from amylopectin), which are separated by amorphous regions (built from amylose and branched points of amylopectin). The hydroxyl groups $(\mathrm{OH})$ of glucose units of starch polymers are accessible to water [3]. For the present low hydration sample of native potato starch we assign the exchange process, quantified through 
$k_{\text {starch-water }}$, to chemical exchange of protons of water molecules with protons of hydroxyl groups of the amylopectin and amylose molecules on the surface of the granule.

\section{Conclusion}

2D time domain NMR results in hydrated starch were used in a two-site exchange model to analyse exchange between protons of the solid starch matrix and protons of water molecules. The exchange rate $\left(86 \mathrm{~s}^{-1}\right)$ found from this analysis clearly indicates that magnetization exchange plays an important role in controlling the observed spin-lattice relaxation in hydrated starch. Thus, any meaningful analysis of NMR spin-lattice relaxation data for water molecule dynamics in this material must include a careful consideration of magnetization exchange effects.

\section{References}

[1] T.Y. Bogracheva, V.J. Morris, S.J. Ring, C.L. Hedley, Biopolymers 45, 323 (1998).

[2] D.J. Gallant, B. Bouchet, P.M. Baldwin, Carbohydr. Polym. 32, 177 (1997).

[3] A.M. Donald, T.A. Waigh, P.J. Jenkins, M.J. Gidley, M. Debet, A. Smith, in: Starch: Structure and Functionality, Eds. P.J. Frazier, A.M. Donald, P. Richmond, Royal Society of Chemistry, Cambridge (UK) 1997, p. 172.

[4] J. Szymońska, F. Krok, Inter. J. Biol. Macromol. 33, 1 (2003).

[5] H.-R. Tang, J. Goward, B. Hills, Carbohydr. Polym. 43, 375 (2000).

[6] D. Le Boltan, Y. Rugraff, C. Martin, P. Colonna, Carbohydr. Res. 308, 29 (1998).

[7] H.T. Edzes, E.T. Samulski, J. Magn. Reson. 31, 207 (1978).

[8] D.A. Oleskevich, N. Ghahramany, W.P. Wȩglarz, H. Peemoeller, J. Magn. Reson. $B$ 113, 1 (1996)

[9] H. Harańczyk, in: On Water in Extremely Dry Biological Systems, Wydawnictwo Uniwersytetu Jagiellońskiego, Kraków 2003, p. 91.

[10] W. Derbyshire, M. van den Bosch, D. van Dusschoten, W. MacNaughtan, I.A. Farhat, M.A. Hemminga, J.R. Mitchell, J. Magn. Reson. 168, 278 (2004). 